\title{
Possible Implication of RNF135 in High Type 1 Neurofibromatosis Tumoral Risk
}

Schwartz $\mathbf{M}^{1 *}$, Heide $\mathrm{S}^{1}$, Benzarti $\mathrm{A}^{1}$, Rordriguez $\mathrm{D}^{2,3}$, Marzin $\mathrm{P}^{1}$, Ader $\mathrm{F}^{1}$, Larbi-Messaoud $\mathrm{S}^{4}$, Belhous $\mathrm{K}^{4}$, Keren $\mathrm{B}^{5}$, Whalen $\mathrm{S}^{1}$, Chantot-Bastaraud $\mathbf{S}^{1}$ and Siffroi JP'

${ }^{1}$ Département de Génétique médicale, Hôpital d'Enfants Armand Trousseau, AP-HP, UPMC, Paris, France

'Unité de Neuropédiatrie et de Pathologie du Développement, Hôpital d'Enfants Armand Trousseau, AP-HP, UPMC, Paris, France

${ }^{3}$ CRMR Neurofibromatoses lle-de-France

${ }^{4}$ Service d'imagerie pédiatrique, Hôpital Necker, APHP, Paris, France

${ }^{5}$ Département de Génétique, Hôpital de la Pitié Salpêtrière, AP-HP, Paris, France

\begin{abstract}
Background: Recurrent type 1 and 2 microdeletions of NF1 (neurofibromatosis, type 1) gene leads to a more severe phenotype than heterozygous point mutations. The associated deletion of RNF135 (Ring Finger protein 135), a flanking NF1 gene, is known to be responsible for the childhood overgrowth syndrome but its implication in tumoral severity has never been proven.
\end{abstract}

Methods and Results: A Single Nucleotide Polymorphisms array $(715 \mathrm{~K})$ was performed in a patient with a very severe form of type 1 neurofibromatosis, as revealed by massive cervico-mediastinal plexiform neurofibromas. It showed a small $126 \mathrm{~kb}$ atypical deletion encompassing only RNF135 and the first exon of NF1.

Conclusion: This observation highlights the possible role of RNF135 in tumoral severity in type 1 neurofibromatosis caused by recurrent type 1 and type 2 microdeletions.

Keywords: Neurofibromatosis; RNF135; Cytogenetics; Atypical deletion; Single nucleotide polymorphism array

\section{Introduction}

About $5 \%$ of type 1 neurofibromatosis cases are caused by different types of recurrent overlapping 17q11.2 microdeletions that encompass several genes including NF1 (Neurofibromatosis, type 1) [1,2]. These recurrent deletions are mediated by repeated regions $N F 1-R E P a$ and NF1-REPc for type 1 deletions (1.4Mb containing 15 genes) [3], by SUZ12 (Suppressor of zeste 12) and its pseudogene for type 2 deletions (1.2Mb containing 14 genes) [4], and by $N F 1-R E P b$ and $N F 1-R E P c$ for the much rarer type 3 deletion (1.0Mb containing 9 genes) $[5,6]$. Many atypical deletions have also been reported [5-7].

A severe neurofibromatosis phenotype, including numerous and early-onset plexiform neurofibromas, as well as malignant peripheral nerve sheath tumors (MPNST), is encountered more preferentially in type 1 and type 2 deleted patients than in those with NF1 point mutations $[3,6,8]$. Deleted patients also present an overgrowth syndrome due to the loss of the RNF135 (Ring Finger protein 135) gene [9]. RNF135 is a NF1 flanking gene which is included in type 1 and type 2 microdeletions but its implication in early-onset tumors is not clearly defined so far. By comparing the expression level of the 16 genes included in the $1.4 \mathrm{Mb}$ deletion in dermal plexiform neurofibromas and in MPNST samples with that in benign dermal neurofibromas, Pasmant et al. [10] demonstrated that both RNF135 and ADAP2 (ArfGAP with dual pleckstrin homology domains 2) were down-regulated in MPNST biopsies and MPNST cell lines. These results suggested that one or both of these two genes were involved in the increased malignant risk observed in NF1 deleted patients.

Here we report the case of a young but severe NF1 patient carrying a non-previously described $126 \mathrm{~kb}$ microdeletion encompassing only the RNF135 gene and the NF1 first exon.

\section{Case Report}

\section{Patient clinical data}

This boy was born at 36 weeks of gestation from non-related Caucasian parents. Pregnancy was marked by a diet-treated gestational diabetes, an over $90^{\text {th }}$ percentile macrosomy at $2^{\text {nd }}$ trimester ultrasound examination and a major hydramnios. Birth parameters were $3590 \mathrm{~kg}$ $\left(>95^{\text {th }}\right.$ percentile) of weight, $51 \mathrm{~cm}\left(95^{\text {th }}\right.$ percentile) of size and $37.5 \mathrm{~cm}$ $\left(>>97^{\text {th }}\right.$ percentile) of head circumference. Neonatal skin examination revealed a few café-au-lait spots and about 10 hamartomas.

At the age of 3 months, acute respiratory distress led to his hospitalization. Clinical examination revealed laryngomalacia, hypersalivation and axial hypotonia with peripheral hypertonia. Size and weight were below average at this time while head circumference was at +2 SD. During follow-up, a persistence of neurological troubles, a chronic stridor and several oxygen desaturation episodes around $85 \%$ were noted. As polysomnography revealed severe obstructive sleep apneas, non-invasive ventilation was introduced during sleep. This latter induced a great improvement of the patient's tonus. Psychomotor development was normal afterwards, but chronic dyspnea remained and an infiltration of cervical and facial soft tissues was noted. Laryngotracheal endoscopy with biopsies was performed and showed a bulky cervical plexiform neurofibroma behind left arytenoid cartilage, leading to clinical diagnosis of type I neurofibromatosis. At the age of 15 months, whole body Magnetic Resonance Imaging (RMI) revealed a massive tumor syndrome with numerous plexiform neurofibromas in the cervical and mediastinal region (Figures 1A and 1B). The patient was included in a therapeutic trial by selective inhibitor of MAPK kinase (MEK) inhibitors at the age of 17 months.

*Corresponding author: Dr. Mathias Schwartz, Département de Génétique médicale, Hôpital d'Enfants Armand-Trousseau, 26 Avenue du Dr. Arnold Netter 75012 Paris, France, Tel: (33) 1447352 80, Fax: (33) 1447352 82; E-mail: mathias.schwartz@gmail.com

Received January 10, 2018; Accepted January 30 2018; Published February 02, 2018

Citation: Schwartz M, Heide S, Benzarti A, Rordriguez D, Marzin P, et al. (2018) Possible Implication of RNF135 in High Type 1 Neurofibromatosis Tumoral Risk. J Mol Genet Med 12: 323 doi:10.4172/1747-0862.1000323

Copyright: (c) 2018 Schwartz M, et al. This is an open-access article distributed under the terms of the Creative Commons Attribution License, which permits unrestricted use, distribution, and reproduction in any medium, provided the original author and source are credited 

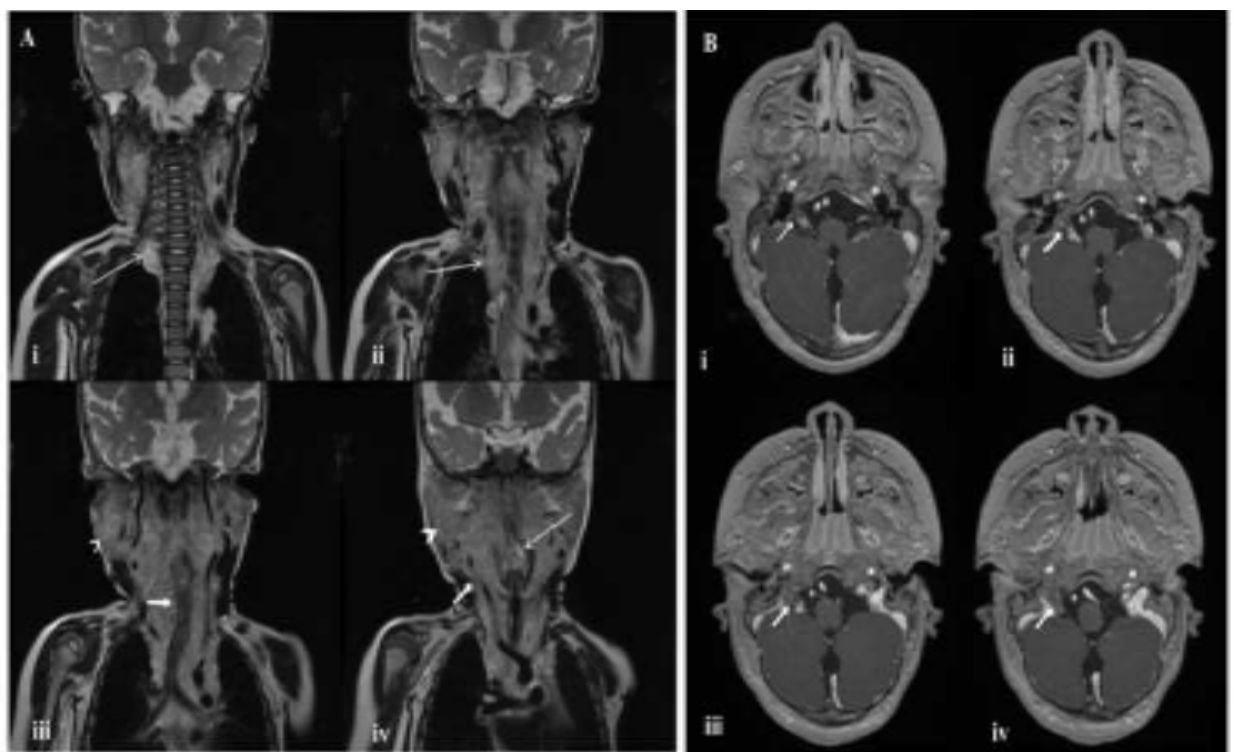

Figure 1: Whole body 3 Tesla Resonance Magnetic Imaging performed at the age of 15 months.

(A) Cervico-thoracic coronal T2 sequence revealing many neurofibromas compressing upper aero-digestive tracks (thick arrow, iii) and repressing carotido-jugular vessels (thick arrow, iv) and submandibular glands, which seep from upper cervico-dorsal paravertebral spaces (thin arrow i,ii) to supraglottic level (thin arrow, iv) through cervical and mediastinal vascular spaces and posteriors cervical spaces. Another neurofibroma from right parotid gland (arrowhead iii, iv) invades right masticator space and right temporo-mandibular joint. (B) Cerebral axial T1 sequence after gadolinium injection showing a $15.4 \mathrm{~mm} \times 9 \mathrm{~mm}$ cerebellopontine angle neurofibroma from cranial nerves IX, X, and XI invading jugular foramen (arrows). A left pinna neurofibroma was also found (not shown).

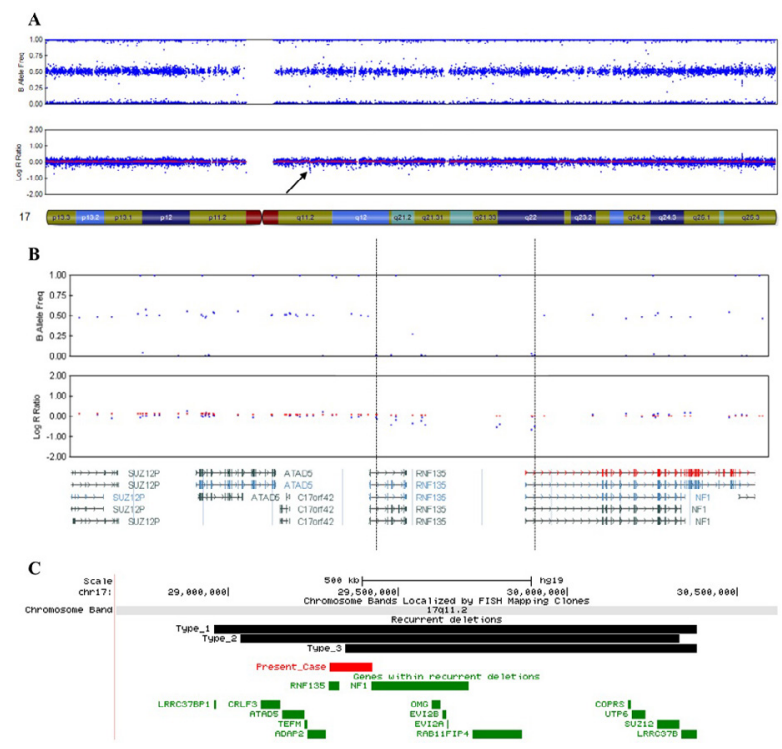

Figure 2: De novo 126kb 17q11.2 microdeletion encompassing RNF135 and the first exon of NF1. arr [hg19] 17q11.2(29,297,595-29,423,989) x1 (A) Single Nucleotide Polymorphisms array (HumanOmniexpress-24, 713K, Illumina $\AA$ ), whole 17 chromosome, black arrow point out the microdeletion. (B) Zoom on deleted region (between the two dotted lines) and the included genes. (C) 17q11.2 recurrent deletions and encompassed genes (genome.ucsc.edu, hg19). Our patient deletion is much smaller than recurrent ones and has, to our knowledge, never been described. Consequent tumoral phenotype is however as severe as type 1 and type 2 recurrent deletions, corroborating thereby the possible implication of RNF135 in tumors invasiveness.

\section{Single nucleotide polymorphisms array analysis}

Single Nucleotide Polymorphisms (SNP) array was performed using the Illumina OmniExpress-24 BeadChip array (713 000 markers, Illumina, San Diego, California, USA). BeadChips were imaged using the Illumina Bead Array reader and data analysis was performed by examination of signal intensity [ $\log \mathrm{R}$ ratio, i.e. $\ln$ (sample copy number/reference copy number)] and allelic composition (B Allele
Frequency) with the copy number variation (CNV) Partition 3.1.6 algorithm in GenomeStudio v.2011.1 (Illumina). Deletion breakpoints were defined as the first and last SNP comprised in the deleted region.

\section{Genetic analysis results}

The karyotype was normal, 46,XY. SNP-array revealed a small $126 \mathrm{~kb}$ microdeletion in the $17 \mathrm{q} 11.2$ region [arr [hg19] 17q11.2 $(29,297,595-29,423,989) \mathrm{x} 1]$ encompassing only RNF135 gene and 
the first exon of NF1 gene (Figures $2 \mathrm{~A}$ and $2 \mathrm{~B}$ ). It was confirmed and visualized by fluorescence in situ hybridization (FISH) using bacterial artificial chromosome clone RP11-229K15. This deletion had never been reported in the literature and it was shown to have occurred de novo since none of the two parents carried it.

\section{Discussion}

Autosomal dominant Neurofibromatosis type 1 (MIM\#162200) has a complete penetrance but shows highly variable expressivity and severity. Type 1 deletions are responsible for a contiguous gene syndrome leading to learning disabilities, facial dysmorphism and childhood overgrowth [6]. These features are also found, but at lower frequencies, in NF1 mutated patients [6]. However, the only well-established genotype-phenotype correlation is the association between type $1^{6}$ or type $2^{8}$ recurrent $17 \mathrm{q} 11$ deletions and an increased tumoral severity when compared to that due to NF1 point mutations. Mantripragada et al. [7] described a 6 years old American patient with café-au-lait spots, freckling and neurofibromas due to a very small $6 \mathrm{~kb}$ atypical deletion, thus suggesting that the loss of the first NF1 exon was sufficient to cause type 1 neurofibromatosis.

We report the occurrence of a very severe tumoral phenotype in a one-year-old boy with neurofibromatosis due to a small $126 \mathrm{~kb}$ deletion encompassing the NF1 first exon and the RNF135 gene. We hypothesized that the severity of our patient's neurofibromas, similar to recurrent type 1 and type 2 longer deletions (Figure 2C), was therefore a consequence of the loss of the RNF135 gene. Indeed, heterozygous loss of function mutations of the neighboring RNF135 gene have been described in dysmorphic patients without neurofibromatosis but presenting with a global overgrowth [9]. It could be linked to an increased cell division rate, thus explaining why loss of RNF135 in deleted NF1 patients could worsen tumoral phenotype. At this point, this is just an assumption as it is very difficult to establish genotypephenotype correlation in a disease with a highly variable expressivity. Describing phenotype of patients with similar deletions could help to conclude about this question.

There is however several arguments pointing to a possible role of RNF135 gene in tumorigenesis. In neurofibromatosis tumors from patients carrying the $1.4 \mathrm{Mb}$ deletion, expression analyses using realtime quantitative reverse-transcription polymerase chain reaction (RTPCR) found a down-regulation of RNF135, suggesting that this gene could be responsible for the increased risk of malignancy observed in deleted NF1 patients [10]. The ubiquitously expressed RNF135 protein contains an E3 ubiquitin ligase domain but its role is poorly known. It has been involved in retroviral infections [11] as well as in some autistic spectrum troubles [12]. RNF135 promotes PTEN and P53 tumor suppressor gene expression since the inactivation of RNF135 increases cell viability, proliferation and invasion in a tongue cancer cell line [13]. Paradoxically, RNF135 seems to have a proliferation promoting action in glioblastoma cells [14]. In this study, the authors suggested that RNF135 could modulate the ERK pathway. Therefore, selective inhibitor of MAPK kinase (MEK) might be of particular interest in 17q11.2 deleted patient. MEK inhibitors have been proposed in NF1related malignant tumors for several years [15-17] and, recently, it has been shown that they have a significant effect on plexiform neurofibromas size [18].

\section{Conclusion}

To our knowledge, the association between MEK inhibitors efficiency and the type of NF1 constitutional abnormality, i.e. microdeletions versus point mutations, has not yet been studied. Our clinical observation supports that RNF135 could promote plexiform neurofibromas development in NF1 deleted patient. However, functional studies will be necessary for a better understanding of its implication in tumorigenesis.

\section{References}

1. Cnossen M (1997) Deletions spanning the neurofibromatosis type 1 gene: Implications for genotype-phenotype correlations in neurofibromatosis type 1? Hum Mutat 9: 458-464.

2. Kluwe $L$ (2004) Screening 500 unselected neurofibromatosis 1 patients for deletions of the NF1 gene. Hum Mutat 23: 111-116.

3. López-Correa (2001) Recombination hotspot in NF1 microdeletion patients Hum Mol Genet 10: 1387-1392.

4. Petek E (2003) Mitotic recombination mediated by the JJAZF1 (KIAA0160) gene causing somatic mosaicism and a new type of constitutional NF1 microdeletion in two children of a mosaic female with only few manifestations. J Med Genet 40: 520-525.

5. Bengesser K, Cooper DN, Steinmann K, Kluweet L (2010) A novel third type of recurrent NF1 microdeletion mediated by nonallelic homologous recombination between LRRC37B-containing low-copy repeats in 17q11.2. Hum Mutat 31: 742-751.

6. Pasmant, E, Sabbagh A, Spurlock G, Laurendeau I, Grillo E, et al. (2010) NF1 microdeletions in neurofibromatosis type 1: From genotype to phenotype. Hum Mutat 31: E1506-E1518.

7. Mantripragada KK (2006) Identification of novel deletion breakpoints bordered by segmental duplications in the NF1 locus using high resolution array-CGH. J Med Genet 43: 28-38.

8. Vogt, J, Nguyen R, Kluwe L, Schuhmann M, Roehl AC, et al. (2011) Delineation of the clinical phenotype associated with non-mosaic type-2 NF1 deletions: two case reports. J Med Case Rep 5: 1-7

9. Douglas J, Cilliers D, Coleman K, Tatton-Brown K, Barker K, et al. (2007) Mutations in RNF135, a gene within the NF1 microdeletion region, cause phenotypic abnormalities including overgrowth. Nat Genet 39: 963-965.

10. Pasmant $E$ (2010) Identification of genes potentially involved in the increased risk of malignancy in NF1-microdeleted patients. Mol Medicine Camb Mass 17: 79-87

11. Oshiumi H, Matsumoto M, Hatakeyama S, Seya T (2009) Riplet/RNF135, a RING finger protein, ubiquitinates RIG-I to promote interferon- $\beta$ induction during the early phase of viral infection. J Biol Chem 284: 807-817.

12. Tastet J, Decalonne L, Marouillat S, Malvy J, Rose-Anne T, et al. (2015) Mutation screening of the ubiquitin ligase gene RNF135 in French patients with autism. Psychiatr Genet 25: 263-267.

13. Jin J, Zhao L, Li Z (2016) The E3 ubiquitin ligase RNF135 regulates the tumorigenesis activity of tongue cancer SCC25 cells. Cancer Med 5: 3140 3146 .

14. Xu Y, Liu Y (2016) RNF135, RING finger protein, promotes the proliferation of human glioblastoma cells in vivo and in vitro via the ERK pathway. Sci Reports 6: 20642.

15. Jessen W, Miller SJ, Jousma E, Wu J, Rizvi TA, et al. (2013) MEK inhibition exhibits efficacy in human and mouse neurofibromatosis tumors. J Clin Invest 123: $340-347$

16. Jousma, E, Rizvi TA, Wu J, Janhofer D, Dombi E, et al. (2015) Preclinical assessments of the MEK inhibitor PD-0325901 in a mouse model of neurofibromatosis type 1. Pediatr Blood Can 62: 1709-1716.

17. Ameratunga, McArthur, Gan H, Cher L (2016) Prolonged disease control with MEK inhibitor in neurofibromatosis type l-associated glioblastoma. J Clin Pharm Ther 41: 357-359.

18. Dombi P (2016) Activity of selumetinib in neurofibromatosis Type 1-related plexiform neurofibromas. New Engl J Medicine 375: 2550-2560. 